\title{
Effect of Humic Acid on Pyrene Removal from Water by Polycation- Clay Mineral Composites and Activated Carbon
}

\author{
Adi Radian ${ }^{\dagger}$ and Yael Mishael ${ }^{*}{ }^{\dagger}$ \\ ${ }^{\dagger}$ The Robert H. Smith Faculty of Agriculture, Food and Environment, The Hebrew University of Jerusalem, Rehovot 76100, Israel \\ Supporting Information
}

ABSTRACT: Pyrene removal by polycation-montmorillonite (MMT) composites and granulated activated carbon (GAC) in the presence of humic acid (HA) was examined. Pyrene, HA, and sorbent interactions were characterized by FTIR, fluorescence and zeta measurements, adsorption, and column filtration experiments. Pyrene binding coefficients to the macromolecules were in the order of PVPcoS (poly-4-vinylpiridine-co-styrene) > HA > PDADMAC (poly diallyl-dimethyl-ammonium-chloride), correlating to pyrene-macromolecules compatibility. Electrostatic interactions explained the high adsorption of HA to both composites $(\sim 100 \%)$, whereas HA adsorption by GAC was low. Pyrene removal by the composites, unlike GAC, was enhanced in the presence of HA; removal by PDADMAC-MMT increased from $\sim 50\left(k_{d}=2.2 \times 10^{3} \mathrm{~kg} / \mathrm{L}\right)$ to $\sim 70 \%\left(k_{d}=2.4 \times 10^{3} \mathrm{~kg} / \mathrm{L}\right)$ in the presence of HA. This improvement was attributed to the adsorption of pyrene-HA complexes. PVPcoS-MMT was most efficient in removing pyrene $\left(k_{d}=1.1 \times 10^{4} \mathrm{~kg} / \mathrm{L},>95 \%\right.$ removal $)$ which was explained in terms of specific $\pi$ donor $-\pi$ acceptor interactions. Pyrene uptake by column filters of GAC reached $\sim 50 \%$ and decreased to $\sim 30 \%$ in the presence of HA. Pyrene removal by the PVPcoS-MMT filter was significantly higher (100-85\% removal), exhibiting only a small decrease in the presence of HA. The utilization of $\mathrm{HA}$ as an enhancing agent in pollutant removal is novel and of major importance in water treatment.

\section{INTRODUCTION}

Polycyclic aromatic hydrocarbons (PAHs) are a group of persistent hydrophobic contaminants that have been detected in aquatic systems all over the world and are included in the US EPA and the EU priority list of pollutants (e.g., benzo(a)pyrene limit in water is $0.0002 \mathrm{mg} / \mathrm{L}){ }^{1,2}$

Fate and transport of hydrophobic organic pollutants (including PAHs) in the environment is strongly affected by interactions with dissolved organic matter (DOM). ${ }^{3-5}$ These interactions depend on both the chemical and structural characteristics of the pollutant and of the DOM and on the characteristics of the environmental system. ${ }^{6}$ Studies have shown that in environmental systems the fate of organic contaminants can vary greatly in terms of adsorption or mobility. Several studies have demonstrated higher solubility and mobility of the pollutants in the presence of DOM. ${ }^{7-9}$ For example, a study on enhanced organic pollutant solubility by DOM demonstrated increased partitioning coefficients into DOM with a decrease in pollutant water solubility and DOM polarity. ${ }^{7}$ On the other hand, other studies have shown that the presence of DOM increased sorption and immobilization of organic pollutants to soil sediments and solid surfaces. ${ }^{9-11}$ RavAcha and Rebhun 1992 suggested that DOM tended to solubilize organic pollutants when the adsorption coefficients (of the DOM) to the clay were relatively low, while adsorption of the pollutants to the clay increased as the DOM adsorption coefficients to the clay increased. ${ }^{9}$ A later study also emphasized this complex behavior; the adsorption of an insecticide to soils decreased in the presence of DOM due to competition for adsorption sites, while the presence of DOM increased the adsorption of the more hydrophobic pollutants. ${ }^{12}$ PAHs tend to exhibit higher mobility in the presence of DOM due to (a) enhanced solubility induced by DOM-pollutant complexation and/or (b) competitive adsorption to the binding sites of the solid surfaces. $6,7,10,12-14$

DOM-pollutant interactions have an important role not only concerning the pollutants' fate in the environment but also in water treatment processes. One of the most common sorbents used for adsorption of PAHs is granulated activated carbon (GAC), but it is well-known that DOM and its derivatives such as humic and fulvic acids significantly reduce the effectiveness of the filtration. ${ }^{15-17}$ Furthermore, DOM in itself is an undesired component in water systems (regulatory levels in drinking water in the US $<80 \mu \mathrm{g} / \mathrm{L}$ ) due to color, taste, and odor, the enhancement of biofilm formation, interactions with $\mathrm{Cl}_{2}$ to form harmful disinfection byproduct, and also due to its interference with water treatment processes (as occurs with GAC). Despite many studies reporting the complexation of DOM with organic pollutants, the exploitation of DOM to

Received: March 11, 2012

Revised: April 23, 2012

Accepted: April 30, 2012

Published: April 30, 2012 
enhance remediation performance has not been systematically investigated. Therefore, the development of sorbents which can simultaneously remove DOM and organic pollutants without decreasing the efficiency of pollutant removal (as occurs with GAC) and in some cases even improving removal is of prime interest.

In the last couple of decades a new class of materials based on polymer adsorption to clay minerals has been developed as sorbents. ${ }^{16,18-20,23}$ In several studies we have demonstrated the superior adsorption of organic pollutants such as herbicides and phenolic compounds to these composites. ${ }^{16,21,22}$ Efficient removal of atrazine by poly-4-vinylpyridine-co-styrene (PVPcoS)-clay composites was demonstrated. ${ }^{16}$ The high binding affinity of atrazine to this composite was explained in terms of compatibility between the herbicide and the polymer. Column filters of the composite removed $93-96 \%$ of atrazine even in the presence of DOM, whereas GAC filters removed $75-83 \%$ which decreased in the presence of DOM to $52-$ $68 \% .^{16}$

Pyrene adsorption to clay minerals has been widely studied. $^{24,25}$ In contrast; its adsorption to clays modified with organic cations (organoclays) has been sparsely evaluated. $\mathrm{Qu}$ et al. 2008, however, have demonstrated enhanced adsorption of PAHs to MMT modified by quaternary ammonium cations, attributed to cation- $\pi$ interactions. ${ }^{26}$

In the current study the removal of pyrene by polycation-clay composites and GAC in the presence of humic acid (HA) was explored. Pyrene was chosen as a model PAH due to its specific volatility and miscibility characteristics, relatively soluble yet hydrophobic. Pyrene adsorption to these composites in the presence of HA remained high and was even enhanced. We suggest the results are due to (I) the composites being designed to specifically bind pyrene, and therefore its adsorption is less likely to be compromised by the presence of HA. Pyrene is a $\pi$ electron donor able to bind specifically to the vinyl-pyridine which is a strong $\pi$-electron acceptor. (II) HA which is negatively charged is efficiently removed by the positively charged composites and (III) the formation of HA-pollutant complexes enhances pyrene adsorption by a cosorption mechanism. The interactions between pyrene, HA, and polycation-clay composites were meticulously investigated. Pyrene uptake with/without HA by the designed composites and by GAC was studied applying suspension (batch) and filtration experiments. Previous studies report the detrimental affect imposed by HA, We suggest the manipulation of the complexation phenomenon to improve PAHs uptake from water, which is a new concept in water treatment technologies.

\section{MATERIAL AND METHODS}

Materials. Wyoming Na-montmorilonite SWy-2 (MMT) was obtained from the Source Clays Repository of the Clay Mineral Society (Columbia, MO); cation exchange capacity (CEC) and specific surface area were $76.4 \mathrm{meq} / 100 \mathrm{~g}$ and 756 $\mathrm{m}^{2} / \mathrm{g}$, respectively. ${ }^{27}$ Poly-4-vinylpiridine-co-styrene - PVPcoS $(\mathrm{MW}=1,200,000-1,500,000 \mathrm{Da}$; PVP-to-S ratio 9:1), poly diallyl-dimethyl-ammonium-chloride - PDADMAC (MW 400,000-500,000 Da), pyrene (98\% purity), and humic acid sodium salt technical grade (HA) were purchased from Sigma Aldrich (Stenheim, Germany). To dissolve PVPcoS, 90\% of the monomers (pyridine) were charged by adding stiochometric concentrations of $\mathrm{H}_{2} \mathrm{SO}_{4}$ (95\% purity). Granular activated carbon (Hydraffin 30N - 0.5-2.5 mm, $900 \mathrm{~m}^{2} / \mathrm{g}$ ) was purchased from Benchmark Ltd. (Israel). Quartz sand (grain size $0.8-1.5 \mathrm{~mm}$ ) was purchased from Shoshani and Weinstein (Israel).

Methods. Fluorescence Measurements; Stern-Volmer Plots. The fluorescence properties of pyrene $(0.05 \mathrm{mg} / \mathrm{L})$ in the presence of PDADMAC, PVPcoS, and HA $(0-24 \mathrm{mg} / \mathrm{L})$ were monitored. Pyrene solutions were prepared in a stock solution of methanol $(1000 \mathrm{ppm})$ and then diluted to the required concentration. The Stern-Volmer fluorescence quenching approach was applied, Stern-Volmer plots were obtained, and the binding constants were calculated according to Gauthier et al. $19866^{28}$ The linear Stern-Volmer plots are based on the assumption that the macromolecules are ideal quenchers; quenching sites are homogeneous, and quenching is a complete and solely dynamic or solely static process. The slopes of the Stern-Volmer plots were calculated as follows: $F_{0} / F_{\text {cor }}=1+k_{b}[$ Macromolecule $(\mathrm{mg} / \mathrm{L})] ; k_{b}$ represents the binding affinity of pyrene to the macromolecules, $F_{0}$ is the fluorescence intensity of pyrene alone in solution, and $F_{\text {cor }}$ is the fluorescence intensity of pyrene after binding to the macromolecule (with inner filter correlation). ${ }^{28}$

In order to properly compare the adsorption results in this study, pyrene adsorption isotherms (ranging in concentrations from 0 to $0.05 \mathrm{mg} / \mathrm{L}$ ) to PVPcoS and HA (for two concentration 3 and $10 \mathrm{mg} / \mathrm{L}$ ) were constructed and Freundlich binding coefficients were obtained. The Freundlich equation relates the concentration of pyrene adsorbed on the surface $\left(C_{\mathrm{ads}}(\mathrm{mg} / \mathrm{kg})\right)$ to the concentration in the solution $\left(C_{\mathrm{eq}}(\mathrm{mg} / \mathrm{L})\right)$ as follows: $C_{\mathrm{ads}}=k_{f}{ }^{*} C_{\mathrm{eq}}{ }^{n} \cdot K_{d}$ coefficients were calculated for the appropriate concentrations- $k_{d}=k_{f}^{*} C_{\mathrm{eq}}{ }^{(n-1)}$. The excitation and emission for pyrene were measured at 273 and $371 \mathrm{~nm}$ respectively, by a fluorescence spectrophotometer (Cary Eclipse, Varian fluorescence spectrophotometer, Agilent technologies). To correct the "inner-filter effect", ${ }^{28} \mathrm{HA}$, PVPcoS, and PDADMAC (0-24 ppm) absorbance was measured by UV-vis spectrophotometry (Thermo Scientific, Evolution 300, Waltham, MA, USA) at the above excitation and emission wavelengths.

Preparation of Composites; PVPcoS and PDADMAC Adsorption on MMT. The polymer-clay composites were prepared as described in our previous studies. ${ }^{16,22,30}$ MMT clay suspension of $0.05 \% \mathrm{w} / \mathrm{w}(0.5 \mathrm{~g} / \mathrm{L}, 4 \mathrm{~mL})$ was added to PDADMAC $(0.01-1 \mathrm{~g} / \mathrm{L})$ or PVPcoS $(0.01-1 \mathrm{~g} / \mathrm{L})$ solutions $(8 \mathrm{~mL})$. The composites were prepared by applying batch experiments using glass tubes $(12 \mathrm{~mL})$ with screw on Teflon caps. The clay-polycation suspension was agitated for $2 \mathrm{~h}$ (equilibrium reached within $1 \mathrm{~h}$ ) using a shaker, suspensions were centrifuged (4000 rpm for $20 \mathrm{~min}$ ), and supernatant was separated. The precipitates were washed and freeze-dried, and the percentage of carbon was measured using a CHNSO analyzer (Thermo-Scientific, Fisons, EA1108). The amount of polymer adsorbed was calculated accordingly. For the suspension and filtration experiments the loadings chosen for the composites PDADMAC-MMT and PVPcoS-MMT were 0.06 clay and $0.07 \mathrm{~g}$ polymer/g, respectively.

Zeta Potential Measurements. The mobilities/zeta potentials of polymer clay composites, PDADMAC-MMT and PVPcoS-MMT (0-2 mmol polymer/g clay), were monitored as a function of HA adsorption using a Zetasizer Nanosystem (Malvern Instruments, Southborough, MA). The different composites were prepared as described, and zeta potentials were measured in a $\sim 0.05 \%$ clay suspension sampled from the top of the vial after settling of $0.5 \mathrm{~min}$. Pyrene solutions (In. Conc. $0.05 \mathrm{mg} / \mathrm{L}$ ) containing $\mathrm{HA}$ (none or an initial 
concentration of $3 \mathrm{mg} / \mathrm{L}$ ) were added to the composites; after $24 \mathrm{~h}$ of agitation and centrifugation (4000 rpm from $20 \mathrm{~min}$ ) the supernatant was discharged, the composites were resuspended in water, and zeta potential was measured.

In addition, HA adsorption was quantified by UV-vis spectrophotometer at a wavelength of $254 \mathrm{~nm}$.

FTIR Measurements. FTIR spectroscopy was used to examine the interactions of HA with the two polycations. Solutions of $10 \mathrm{mg} / \mathrm{L}$ PDADMAC and PVPcoS were mixed with HA in concentrations of $5 \mathrm{mg} / \mathrm{L}(1: 1 \mathrm{w} / \mathrm{w}$ HA/Polymer ratio) and $10 \mathrm{mg} / \mathrm{L}(2: 1 \mathrm{w} / \mathrm{w})$ in $250 \mathrm{~mL}$ centrifuge tubes for 4 $\mathrm{h}$ and then freeze-dried. The spectra were obtained by pellets from dried HA, PVPcoS, PDADMAC, and complexes prepared (PDADMAC+HA and PVPcoS+HA) mixed with $\mathrm{KBr}$ and were recorded at room temperature in the range of $500-4000 \mathrm{~cm}^{-1}$ using a FTIR spectrometer (Nicolet Magna-IR-550, Madiso WI).

Adsorption of Pyrene and Humic Acid on Polymer-Clay Composites. Adsorption of Humic Acid on Polymer-Clay Composites. HA solutions ranging from 1 to $20 \mathrm{mg} / \mathrm{L}$ were added (no head space) to glass tubes $(12 \mathrm{~mL})$ containing PDADMAC-MMT (0.055 g/L), PVPcoS-MMT (0.055 g/L) composites, and MMT $(0.5 \mathrm{~g} / \mathrm{L})$ or GAC $(0.5 \mathrm{~g} / \mathrm{L})$. The samples were agitated overnight $(24 \mathrm{~h})$ and centrifuged (4000 rpm from $20 \mathrm{~min}$ ), and the supernatant was separated and filtered through a $0.45 \mu \mathrm{m}$ Acrodisc syringe filters before analysis by UV-vis spectrophotometer at a wavelength of 254 $\mathrm{nm}$.

Adsorption of Pyrene in the Presence of Humic Acid by the Polymer-Clay Composites and Unmodified MMT. Pyrene $(0-0.075 \mathrm{mg} / \mathrm{L})$ and $\mathrm{HA}(0-10 \mathrm{mg} / \mathrm{L})$ solutions were added to glass tubes $(12 \mathrm{~mL})$ containing PDADMACand PVP-co-S-MMT composites. The samples were agitated for $0.125-24 \mathrm{~h}$ in the presence of $3 \mathrm{mg} / \mathrm{L} \mathrm{HA}$ for the kinetic experiments and for $24 \mathrm{~h}$ in equilibrium experiments. The samples were centrifuged (4000 rpm from $20 \mathrm{~min}$ ), and the supernatants were separated for analysis; pyrene by fluorescence spectroscopy and HA concentrations by UV-vis spectrophotometer.

All analyses were performed in triplicates and compared with control tubes containing pyrene to account for any loss during the course of the experiment. The pyrene concentrations for samples containing HA were recalculated by the Stern-Volmer correlation and compared with separate calibration curves for each concentration.

Pyrene and HA Removal by Filtration with Composites and GAC Filters. Pyrene $(0.06 \mathrm{mg} / \mathrm{L}$ in tap water) filtration, with and without HA $(5 \mathrm{mg} / \mathrm{L})$, was studied. Glass columns of $22 \mathrm{~cm}$ in length and of $1.5 \mathrm{~cm}$ in diameter were filled with $65 \mathrm{~g}$ of sand mixed with $0.65 \mathrm{~g}$ composites $(1: 100 \mathrm{w} / \mathrm{w})$ or with a layer of $0.65 \mathrm{~g}$ of GAC. Preliminary experiments showed no significant difference in pollutant removal by filtration through columns filled with a layer of GAC or with it mixed with the sand. The composites were mixed with excess quartz sand to enable high flow through the column. The sand was thoroughly washed in distilled water and dried at $105{ }^{\circ} \mathrm{C}$ for $24 \mathrm{~h}$. Nonwoven polypropylene geo-textile (Markham Culverts Ltd., Papua New Guinea) was placed on both ends of the column to prevent exit of the sand from the column. The pyrene stock solutions $(5 \mathrm{~L})$ were passed through each of the filters at a flow rate of $7 \mathrm{~mL} / \mathrm{min}$. The eluting solutions were collected every $70 \mathrm{~min}(0.5 \mathrm{~L})$ and analyzed by UV-vis spectrophotometer for the $\mathrm{HA}$ and fluorescence spectrophotometer for the pyrene.
The pyrene concentrations for samples containing HA were recalculated by the Stern-Volmer correlation and separate calibration curves for each HA-pyrene concentration.

\section{RESULTS AND DISCUSSION}

The removal of pyrene in the presence of HA by two polymer clay composites was investigated. One composite incorporated PDADMAC, a highly charged quaternary ammonium polycation adsorbed on MMT $(0.06 \mathrm{mmol} / \mathrm{g})$ and the second composite, incorporated PVPcoS, a copolymer containing styrene and positively charged pyridine rings (ratio of 1:9) adsorbed on MMT $(0.065 \mathrm{mmol} / \mathrm{g})$. It was hypothesized that pyrene would show higher affinity to the PVPcoS composites as a result of structural compatibility (enabling $\pi-\pi$ interactions), HA (negatively charged) would show high affinity to both composites due to strong electrostatic interactions, and pyrene would complex with HA and enhance (cosorption mechanism) pyrene adsorption to the composites. Enhancement of pyrene adsorption in the presence of HA would be more pronounced in the case of PDADMAC composites compared with PVPcoS composites due to direct low and high pyrene adsorption on the composites, respectively.

Pyrene Interactions with Polycations or HA; Fluorescence Measurement. Interactions between pyrene and the three macromolecules (PDADMAC, PVPcoS or HA) in solution were characterized. The binding of pyrene was analyzed by two models, Stern-Volmer plots ${ }^{28}$ and Freundlich adsorption isotherms, to determine the relative affinity of pyrene (Supporting Information (SI) Figure S1A-B). These models have been implemented in studies on HA interactions (and other fractions of DOM), ${ }^{25,28,30-32}$ and in the current study they were expanded to characterize interactions with polycations. Linear Stern-Volmer plots were observed for all three macromolecules. Freundlich isotherms were derived for pyrene binding to HA and PVPcoS (binding to PDADMAC was not calculated since the interactions observed by SternVolmer were low) and coefficients were fitted (SI Table S1).

Pyrene binding affinities calculated from the Stern-Volmer $\left(k_{b}\right)$ plots and Freundlich isotherms $\left(k_{d}\right)$ were in the order of PVPcoS > HA > PDADMAC. The very high affinity between pyrene and PVPcoS $\left(k_{b} 4.5 \times 10^{4} \mathrm{~L} / \mathrm{kg}\right.$ and $\left.k_{d} 1.2 \times 10^{5} \mathrm{~L} / \mathrm{kg}\right)$ and high affinity to HA $\left(k_{b} 3.9 \times 10^{4} \mathrm{~L} / \mathrm{kg}\right.$ and $k_{d} 6 \times 10^{4} \mathrm{~L} /$ $\mathrm{kg}$ ) were explained by the degree of structure compatibility (aromatic nature) between pyrene and the macromolecules, high in the case of PVPcoS and moderate for HA. This compatibility results in $\pi$ donor- $\pi$ acceptor interactions; pyrene is a $\pi$-electron donor able to bind specifically to the vinylpyridine which is a strong $\pi$-electron acceptor, and these interactions are not present in the case of PDADMAC explaining the lower binding affinity to this polymer $\left(k_{b} 9.9\right.$ $\times 10^{3} \mathrm{~L} / \mathrm{kg}$ ). The $k_{b}$ and $k_{d}$ constants for pyrene binding to HA are in the same magnitude as coefficients previously reported. $^{28,31,32}$

PDADMAC and PVPcoS Interactions with HA; FTIR Measurements. FTIR spectra of PDADMAC- and PVPcoSHA complexes $(10 \mathrm{mg} / \mathrm{L}$ polycation with 5 or $10 \mathrm{mg} / \mathrm{L} \mathrm{HA})$ were compared to those of pure $\mathrm{HA}$ and polycations spectra (SI Figure S2). The pure HA spectrum indicated characteristic bands at 3430, 2870-2960, 1580, 1390, and 1030-1100 $\mathrm{cm}^{-1} \cdot 33,34$ The bonds at the range of $2870-2960 \mathrm{~cm}^{-1}$ correspond to $\mathrm{CH}_{2}-\mathrm{CH}_{3}$ stretching; the bands at $1580 \mathrm{~cm}^{-1}$ and $1390 \mathrm{~cm}^{-1}$ are indicative of $\mathrm{COO}^{-}$asymmetric and symmetric stretching, respectively. The $\mathrm{COOH}$ vibrations at 
$1720 \mathrm{~cm}^{-1}$ were not present since the $\mathrm{HA}$ was in a salt form. ${ }^{33,34}$ The lower range bands $1030-1100 \mathrm{~cm}^{-1}$ correspond to alcohol and ethers stretching. PDADMAC spectrum showed characteristic peaks at 3460, 2800-3000, 2084, 1647, 1480, and $970-1000 \mathrm{~cm}^{-1} .22$ The band at $2084 \mathrm{~cm}^{-1}$ is attributed to the quaternary ammonium chloride bond; ${ }^{35}$ this vibration showed a slight shift at the low concentration of $\mathrm{HA}$ and at higher HA concentration diminished significantly. We suggest that this behavior is a result of the formation of electrostatic interactions between the quaternary ammonium and the negatively charged $\mathrm{HA}^{22,36,37}$ PVPcoS showed distinctive peaks at 3050, 3000$2800,1950,1450-1600,1200-1210,1000$, and $800-680$ $\mathrm{cm}^{-1}$. The band at $1950 \mathrm{~cm}^{-1}$ corresponds to the charged ammonium on the pyridine; this vibration disappeared as well upon HA-polymer complexation. The aromatic moieties are responsible for the bands at 3050, 1450-1600, and 680-800 $\mathrm{cm}^{-1}$. Small shifts in the aromatic vibration especially in the fingerprint region were observed upon complexation (546-683 $\left.\mathrm{cm}^{-1}\right)$. These shifts may be due to $\pi-\pi$ interactions between aromatic moieties of the HA and the phenyl groups of the polycation. $^{38}$ The FTIR measurements support our hypothesis that the interactions between the polycations and the HA are mainly electrostatic, and in the case of PVPcoS $\pi-\pi$ interactions occur as well.

PDADMAC-MMT and PVPcoS-MMT Interactions with HA and Pyrene; Zeta Potential Measurements. FTIR measurements suggested that the interactions between HA and the two polymer-clay composites are mainly electrostatic; therefore, upon HA adsorption zeta potential is expected to decrease (less positive). Zeta potential of PDADMAC-MMT and PVPcoS-MMT at varying polymer loadings (0-2 mmol polymer/g clay) was monitored upon $\mathrm{HA}(3 \mathrm{mg} / \mathrm{L})$ adsorption (Figure 1A, B). Negatively charged unmodified MMT (-45 $\mathrm{mV})$ did not adsorb the negatively charged HA $(-15 \mathrm{mV})$.

The initial zeta potential of PDAMAC-MMT composites ranged from $-30 \mathrm{mV}$ for a loading of $0.225 \mathrm{mmol} / \mathrm{g}$ (below $\mathrm{CEC})$ and reached $+60 \mathrm{mV}$ at a loading of $2 \mathrm{mmol} / \mathrm{g}(250 \%$ of the CEC) as previously reported. ${ }^{22,30}$ Charge neutralization was reached at a polymer loading of $0.3 \mathrm{mmol}$ polymer/g clay, below the CEC, which was explained in terms of MMT surface screening by the highly charged polycation extending into the solution even at loadings lower than stochiometric neutralization. ${ }^{22,30}$ The adsorption of $\mathrm{HA}$ to the negatively charged composite (prior to surface charge neutralization) was very low $(<10 \%)$. At a polymer loading of $0.5 \mathrm{mmol} / \mathrm{g}$ PDADMAC, zeta potential was $+27.5 \mathrm{mV}$ and $\mathrm{HA}$ adsorption was complete. At higher PDADMAC loadings $(1 \mathrm{mmol} / \mathrm{g})$ a decrease in HA adsorption was apparent despite the positively charged composite. This was explained in terms of aggregation caused by HA bridging between composites which decreased accessible surface area. 22

PVPcoS-MMT composites showed similar behavior; initial zeta potential of the composites ranged from $-30 \mathrm{mV}$ for 0.4 $\mathrm{mmol} / \mathrm{g}$ loading ( $50 \%$ of $\mathrm{CEC}$ ) reaching $+30 \mathrm{mV}$ for a loading of $200 \%$ of CEC ( $1.7 \mathrm{mmol}$ polymer/g). As with PDADMACMMT composites the HA adsorption was complete for the positively charged composites $(>0.65 \mathrm{mmol} / \mathrm{g})$. Insignificant aggregation was observed in the case of PVPcoS-MMT composites. The zeta potential of the composites decreased with the adsorption of HA; however, the decrease was not as significant in the case of the PDADMAC-MMT composite due to the lower charge density on the composites' surface.
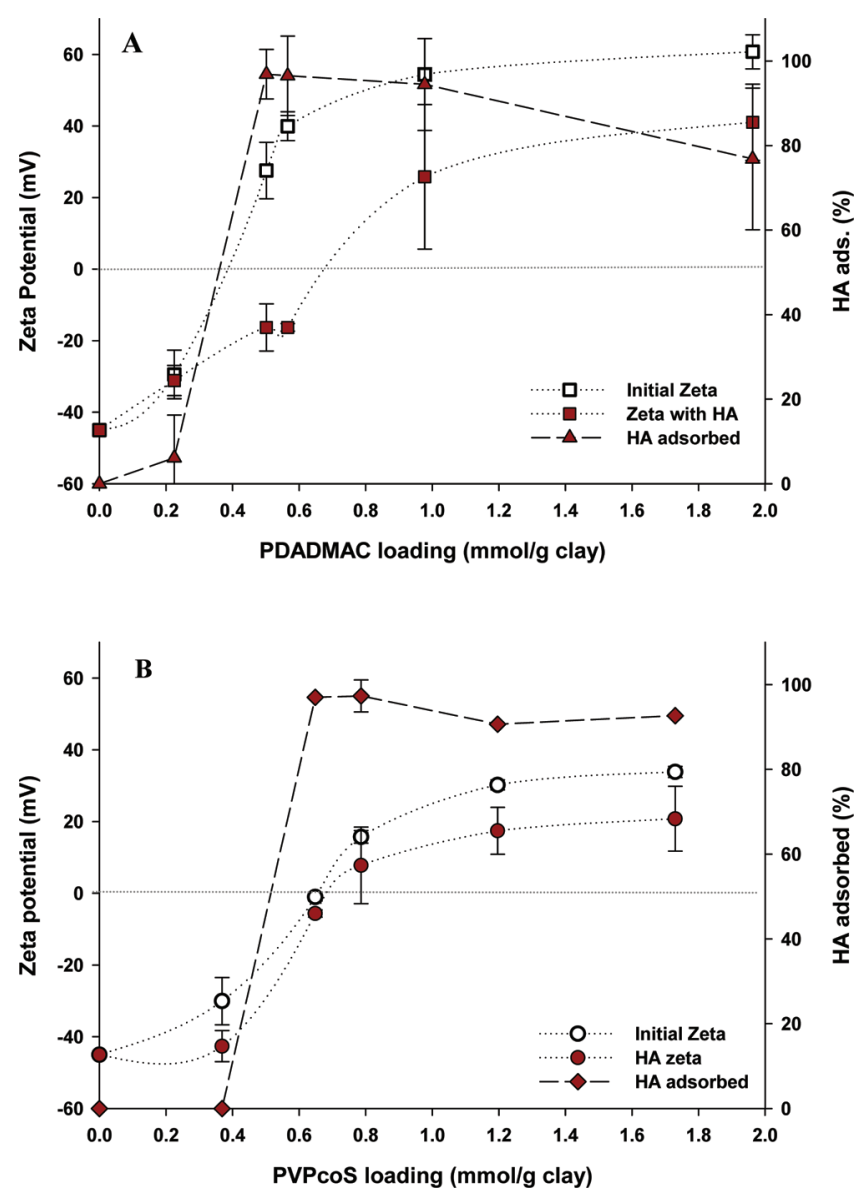

Figure 1. Zeta potential and adsorption of HA (3 ppm) on A. PDADMAC-MMT $(0-2 \mathrm{mmol} / \mathrm{g})$ and B. PVPcoS $(0-1.7 \mathrm{mmol} / \mathrm{g})$.

The composites which obtained maximum HA adsorption were chosen to be further explored, PDADMAC-MMT $(0.5$ $\mathrm{mmol} / \mathrm{g}, 0.06 \mathrm{~g} / \mathrm{g})$ and PVPcoS-MMT $(0.65 \mathrm{mmol} / \mathrm{g}, 0.07 \mathrm{~g} /$ g).

HA Adsorption on PDADMAC-MMT, PVPcoS-MMT, and GAC. The adsorption of HA $(0-10 \mathrm{mg} / \mathrm{L})$ by the two chosen composites and by GAC was studied (Figure 2). The $d$ spacing was also measured upon HA adsorption; however, no increase in the spacing was observed, suggesting that the HA did not enter the interlayers of the composites. The adsorption by GAC decreased as HA concentrations increased from $80 \%$ at $1 \mathrm{mg} / \mathrm{L} \mathrm{HA}$ to less than $2 \%$ adsorption at $10 \mathrm{mg} / \mathrm{L} \mathrm{HA}$. The

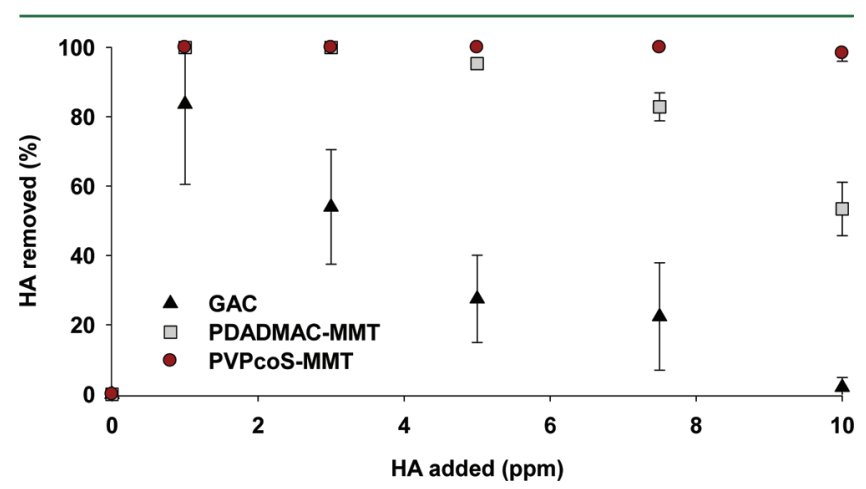

Figure 2. HA (0-10 mg/L) removal by PDADMAC-MMT, PVPcoSMMT, and GAC (0.5 g sorbent/L). 
uptake of HA by the PDADMAC-MMT composite was high (100\%) at low HA concentration but decreased to $53 \%$ adsorption at $10 \mathrm{mg} / \mathrm{L}$; in contrast, $\mathrm{HA}$ adsorption by the PVPcoS-MMT composite was complete even at high HA concentrations. Electrostatic interaction occurs between the HA and both composites, but in the case of PVPcoS-MMT there are $\pi-\pi$ interactions in addition as suggested by the FTIR measurements.

Pyrene Adsorption on Polycation Composites and GAC in the Presence of HA. Adsorption of Pyrene on PDADMAC-MMT, PVPCOS-MMT, and Unmodified MMT in the Presence of $H A$. To elucidate the effects of HA on pyrene adsorption by the different composites, pyrene adsorption to unmodified MMT, PDADMAC-MMT, and PVPcoS-MMT composites was measured in the presence of HA $(0-10 \mathrm{mg} / \mathrm{L})$ (Figure 3). Pyrene adsorption to unmodified MMT which was

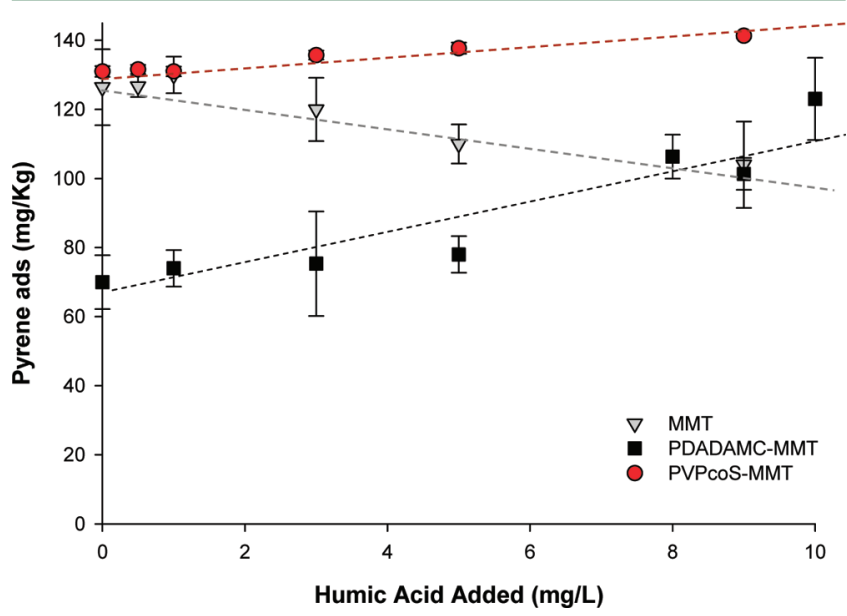

Figure 3. Pyrene adsorption $(0.05 \mathrm{mg} / \mathrm{L})$ by unmodified MMT, PDADMAC-MMT, and PVPcoS-MMT (0.5 g sorbent/L) in the presence of $\mathrm{HA}(0-10 \mathrm{mg} / \mathrm{L})$.

relatively high $(85 \%)$ in comparison to the literature ${ }^{25,39-42}$ was attributed to interactions with the siloxan surfaces, cation- $\pi$ interactions or energetic gain due to the release of water molecules. $^{25}$

Unmodified MMT $(\xi=-45 \mathrm{mV})$ did not bind HA $(\xi=-15$ $\mathrm{mV}$ ) under the conditions set; therefore, pyrene adsorption to MMT decreased upon an increase in HA concentrations which complexes with pyrene-enhancing its solubility. This indicates that pyrene-HA interactions are stronger than those of pyreneMMT. In contrast, the presence of $\mathrm{HA}$ enhanced pyrene adsorption by the polycation-clay composites. The adsorption of pyrene to PDADMAC-MMT was poor at low HA concentrations $(<50 \%)$ as predicted by the Stern-Volmer plots but improved as HA concentrations increased reaching $\sim 70 \%$ removal. This behavior can be attributed to a cosorption (or sweeping) mechanism caused by the HA-pyrene complex adsorbing on the external surface of the composite. The PVPcoS-MMT composite adsorbed pyrene efficiently due to molecular compatibility, as predicted by the fluorescence measurements (Stern-Volmer and Freundlich), and a slight increase in the pyrene adsorption was apparent $(90 \%$ at $0 \mathrm{mg} / \mathrm{L}$ to $95 \%$ at $10 \mathrm{mg} / \mathrm{L} \mathrm{HA}$ ). This was also attributed to cosorption, however, since the pyrene adsorption on the composite is so high the phenomenon is less pronounced.

Kinetic Measurements of Pyrene Adsorption on Polycation Composites and GAC in the Presence of HA. The kinetics of pyrene adsorption by the composites in comparison to GAC was studied in suspension with and without HA (Figure 4) and were found to be in agreement with the equilibrium studies (Figures 3 and 5). The PVPcoS-MMT composite adsorbed pyrene most efficiently, and equilibrium was reached within $2 \mathrm{~h}$ while its adsorption by PDADMAC-MMT was relatively poor and equilibrium was reached within $4 \mathrm{~h}$. Pyrene adsorption to GAC was efficient ( $92 \%)$; however, equilibrium was reached within $24 \mathrm{~h}$. As previously reported, the adsorption kinetics to GAC is slow due to molecular diffusion within the closed, irregular-shaped micropores, while the ordered, semiopened layered structure of clay composites enhances adsorption kinetics. $^{23}$

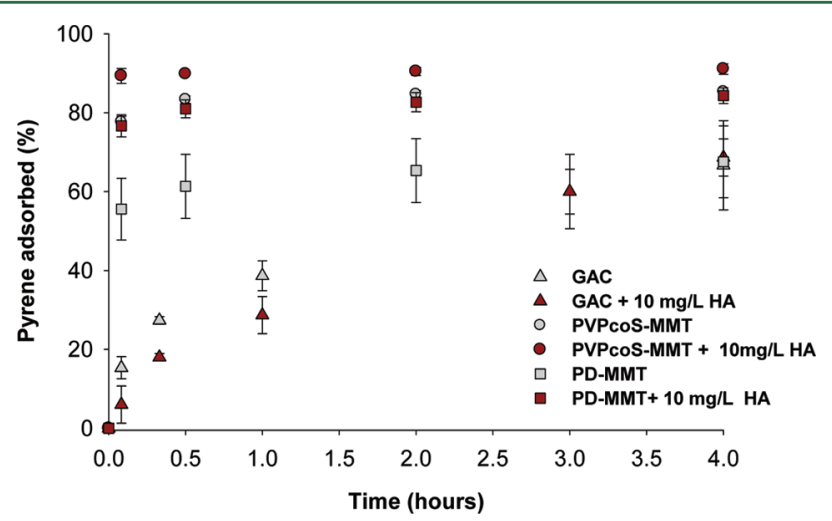

Figure 4. Adsorption kinetics of pyrene $(0.05 \mathrm{mg} / \mathrm{L})$ with and without HA $(10 \mathrm{mg} / \mathrm{L})$ by PDADMAC-MMT, PVPcoS-MMT, and GAC (0.5 g sorbent/L).

The presence of HA enhanced pyrene adsorption and hastened equilibrium (shortened to $0.5 \mathrm{~h}$ ), on the two polycation clay composites. These trends were more pronounced in the case of PDADMAC-MMT due to the low affinity of pyrene to the composite. Conversely, the presence of $\mathrm{HA}$ in the case of GAC decreased pyrene adsorption significantly and hindered equilibrium supporting our findings (Figure 4) that HA adsorption to GAC is low.

Adsorption Isotherms of Pyrene to MMT, PDADMAC-MMT, and PVPCOS-MMT in Equilibrium in the Presence of $H A$. Adsorption isotherms of pyrene to unmodified MMT and to the two composites in the presence of 0 and $3 \mathrm{mg} / \mathrm{L} \mathrm{HA}$ were obtained (SI Figure S3), and the fitted Freundlich coefficients are listed in Table 1. Binding coefficient were in the order of PVPcoS-MMT (HA) > PVPcoS-MMT > MMT > MMT (HA) $>$ PDADMAC-MMT (HA) > PDADMAC-MMT. The trends observed from the isotherms were consistent with the former findings (Figures 3 and 4); pyrene adsorption to the

Table 1. Freundlich Coefficients from Pyrene Adsorption Isotherms (0.05 ppm) to MMT, PDADMAC-MMT, and PVPcoS-MMT without and in the Presence of HA (3 ppm)

\begin{tabular}{lllll}
\multicolumn{1}{c}{ adsorbent } & \multicolumn{1}{c}{$k_{f}$} & $n$ & $R^{2}$ & $\begin{array}{c}k_{d}(\mathrm{~L} / \mathrm{kg}) \\
(0.04 \mathrm{mg} / \mathrm{L})\end{array}$ \\
PVPcoS-MMT + HA & $8 \times 10^{3}$ & 0.76 & 0.9789 & $1.7 \times 10^{4}$ \\
PVPcoS-MMT & $5.5 \times 10^{3}$ & 0.77 & 0.9991 & $1.1 \times 10^{4}$ \\
MMT & $1.7 \times 10^{3}$ & 0.65 & 0.999 & $5.1 \times 10^{3}$ \\
MMT+HA & $7.1 \times 10^{2}$ & 0.54 & 0.9995 & $3.3 \times 10^{3}$ \\
PD-MMT + HA & $1 \times 10^{3}$ & 0.74 & 0.9826 & $2.4 \times 10^{3}$ \\
PD-MMT & $9.5 \times 10^{2}$ & 0.74 & 0.9919 & $2.2 \times 10^{3}$
\end{tabular}


composites was higher in the presence of $\mathrm{HA}$, whereas adsorption of pyrene to the unmodified MMT in the presence of HA decreased. Pyrene adsorption to PVPcoS-MMT was exceptionally high with and without $\mathrm{HA}\left(k_{d}=1.79 \times 10^{4}\right.$ and $k_{d}$ $=1.15 \times 10^{4} \mathrm{~L} / \mathrm{kg}$, respectively), and the coefficients $\left(k_{d}\right)$ calculated were a magnitude higher than the ones previously reported for organo-clays (MMT modified by quaternary ammonium) $(100-1100 \mathrm{~L} / \mathrm{kg}) .{ }^{28}$ However, the Freundlich sorption coefficients for pyrene adsorption to HA in solution calculated from the fluorescence experiments $\left(3-6 \times 10^{4} \mathrm{~L} /\right.$ $\mathrm{kg}$ ) were significantly larger than the coefficients calculated for both the unmodified MMT and composites. These results imply that HA-pyrene complexation was favorable which resulted in enhanced pyrene adsorption via its complexation with HA to the composites and respectively compromised pyrene adsorption to the unmodified MMT and to GAC.

Pyrene Filtration with/without HA by Composites and GAC Columns. The removal of pyrene $(0.065 \mathrm{mg} / \mathrm{L})$ with and without HA by the composites and GAC filtration columns was examined (Figure 5A-C). HA removal by the sorbent filters demonstrated the same trend as in suspension (Figure 2). PDADMAC-MMT and PVPcoS-MMT exhibited high HA removal (Figure 5A) from the solution due to the strong electrostatic interactions (93-86 and 100\%, respectively). PVPcoS-MMT showed complete removal even after the passage of $10 \mathrm{~L}$ (equivalent to 625 pore volumes) (results not shown) which was explained in terms of $\pi-\pi$ interaction in addition to electrostatic ones as observed by FTIR measurements. HA removal by the GAC columns was the lowest (70$50 \%)$.

PDADMAC-MMT showed relatively poor uptake of pyrene with/without HA (Figure 5B); however, due to the poor pyrene removal the increase in pyrene adsorption by a cosorption mechanism in the presence of HA is noticeable. It should be noted that since HA adsorption was nearly complete (Figure5A) and pyrene removal was not efficient, a large fraction of the pyrene does not complex with HA (under these experimental conditions).

Pyrene uptake by the GAC filter (Figure 5c) decreased from $90 \%$ to $\sim 60 \%$ after passage of only $0.5 \mathrm{~L}$ and stayed relatively low ( $\sim 55 \%$ removal). Furthermore, pyrene removal in the presence of HA dropped to $\sim 30 \%$ after the passage of $0.5 \mathrm{~L}$ solution. The reduction of pollutant uptake by GAC in the presence of $\mathrm{HA}$ has been reported $^{16,17,43}$ and has been previously explained by blockage of the GAC adsorption sites; however, the current results suggest a different explanation. High complexation of pyrene with HA as shown by the adsorption coefficients from the fluorescence experiments preserves the pyrene in the solution and since GAC exhibits poor removal of $\mathrm{HA}$ and consequently of the HApyrene complex, pyrene uptake is compromised.

In agreement with the suspension experiments pyrene uptake was the most efficient by PVPcoS-MMT without and with HA (100-88\% and $100-75 \%$, respectively) (Figure 5C). The presence of HA caused a small decrease in pyrene uptake unlike the slight increase observed in the batch experiments (Figure 3).This trend is explained in terms of kinetic competition; the electrostatic adsorption of HA and HA-pyrene to the composite may be faster than that of the free pyrene (undetectable by the suspension kinetic measurements in which the fastest measurement was after $15 \mathrm{~min})$. The adsorption process in filtration (7 $\mathrm{mL} / \mathrm{min}$ ) is much faster and may give the HA a kinetic
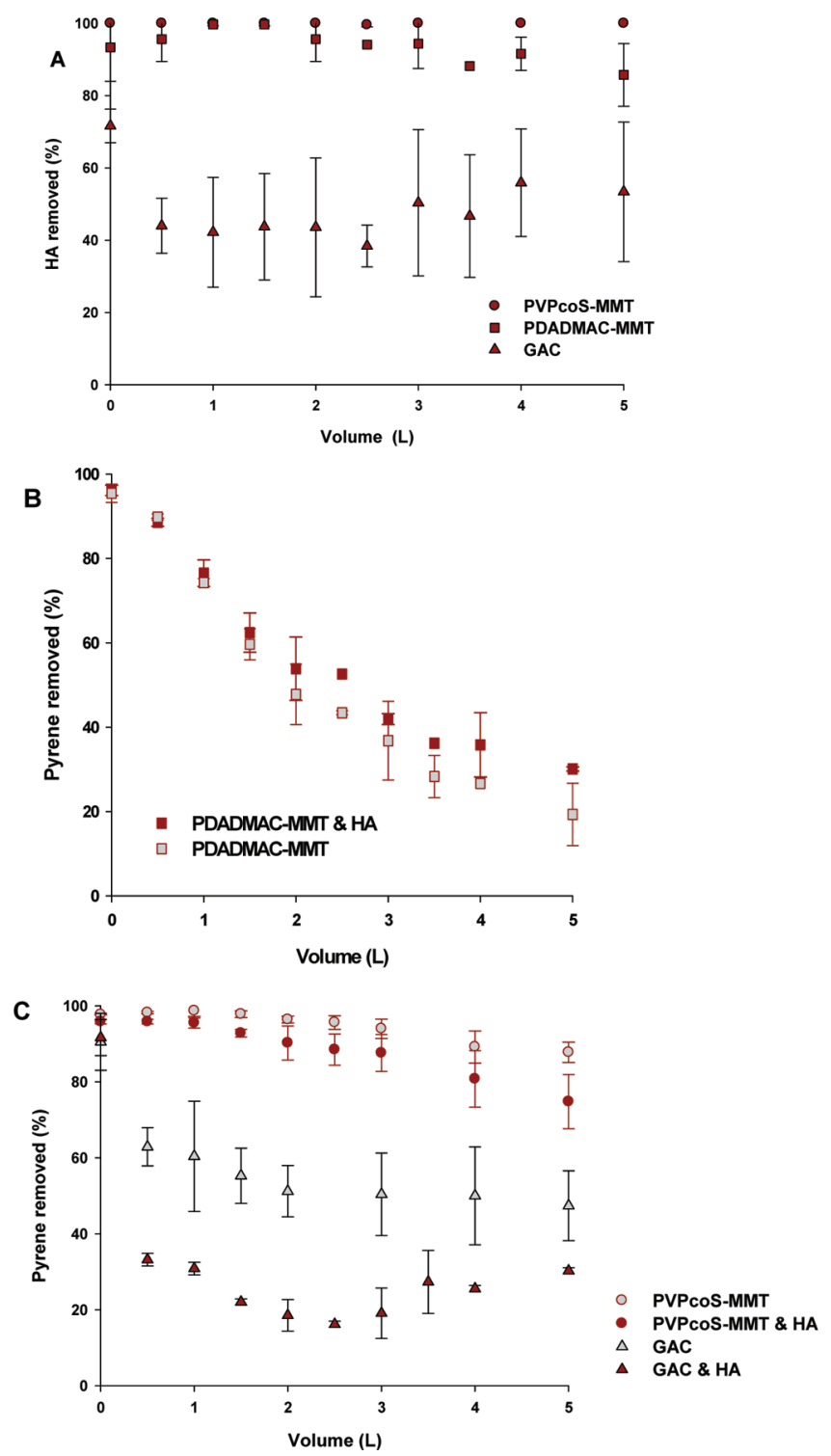

Figure 5. A. Removal of $\mathrm{HA}(5 \mathrm{mg} / \mathrm{L})$ by filtration columns of PVPcoS-MMT, PDADMAC-MMT, and GAC (mixed with sand $(1: 100 \mathrm{w} / \mathrm{w}))$. B. Removal of pyrene $(0.065 \mathrm{mg} / \mathrm{L})$ and pyrene in the presence of HA $(5 \mathrm{mg} / \mathrm{L})$ by PDADMAC-MMT column filter. C. Removal of pyrene $(0.065 \mathrm{mg} / \mathrm{L})$ and pyrene in the presence of HA (5 $\mathrm{mg} / \mathrm{L}$ ) by PVPcoS-MMT or GAC column filters.

advantage over the pyrene which will cause the occupation of adsorption sites reducing noncomplexed pyrene adsorption.

To conclude, pyrene-HA complexation coefficients as calculated by Stern-Volmer and Freundlich models were a magnitude higher than complexation to the composites and unmodified MMT. This complexation improved pyrene adsorption by polycation-clay composites due to the strong electrostatic interactions between the complexed $\mathrm{HA}$ and the composite. In contrast, pyrene removal by GAC was suppressed due to low HA-pyrene complex removal and not solely due to competition on the adsorption sites as previously reported. Filtration and suspension experiments demonstrated the extremely efficient removal of pyrene, even in the presence of $\mathrm{HA}$, by the PVPcoS-MMT composite which was explained in terms of specific interactions between the pollutant and the polymer. We suggest that polymer-clay composites can be design to specifically bind a variety of target pollutants and 
remain highly efficient even in the presence of HA. Furthermore, the novel utilization of $\mathrm{HA}$ as an enhancing agent in pollutant removal is of major importance in water removal technologies.

\section{ASSOCIATED CONTENT}

\section{S Supporting Information}

Stern-Volmer and Freundlich plots from fluorescence measurements (Figure S1 A-B and Table S1), FTIR spectra (Figure S2), and pyrene adsorption isotherms by composites and MMT (Figure S3). This material is available free of charge via the Internet at http://pubs.acs.org.

\section{AUTHOR INFORMATION}

\section{Corresponding Author}

*Phone: 972-8-948-9171. Fax: 972-8-948-9856. E-mail: mishael@agri.huji.ac.il. Corresponding author address: Dept. Soil \& Water Sciences, R.H. Smith Faculty of Agriculture, Food \& Environment, Hebrew University of Jerusalem, Rehovot 76100, Israel.

\section{Notes}

The authors declare no competing financial interest.

\section{ACKNOWLEDGMENTS}

This research was supported by The Bi-National (Israel-US) Foundation (application -\#2010438) and by the EU program ENPI (I-B/2.1/1049).

\section{REFERENCES}

(1) Manoli, E.; Samara, C. Polycyclic aromatic hydrocarbons in natural waters: sources, occurrence and analysis. TrAC Trends Anal. Chem. 1999, 18 (6), 417-428.

(2) Samanta, S. K.; Singh, O. V.; Jain, R. K. Polycyclic aromatic hydrocarbons: environmental pollution and bioremediation. Trends Biotechnol. 2002, 20 (6), 243-248.

(3) Frimmel, F. H.; Abbt-Braun, G.; Heumann, K. G.; Hock, B.; Ludmann, H.-D.; Spiteller, M. Refractory Organic Substances in the Environment; Wiley-VCH: Weinheim, 2002.

(4) Frimmel, F. H.; Christman, R. F. In Humic substances and their role in the environment; John Wiley \& Sons: New York, 1988.

(5) Spitzy, A.; Leenheer, J. Dissolved organic matter in rivers. In Biogeochemistry of Major World Rivers; Degens, E. T., Kempe, S., Richey, J. E., Eds.; John Wiley \& Sons: New York, 1991; pp 213-232.

(6) Kordel, W.; Dassenakis, M.; Lintelmann, J.; Padberg, S. The importance of natural organic material for environmental processes in waters and soils. Pure Appl. Chem. 1997, 69 (7), 1571-1600.

(7) Chiou, C. T.; Malcolm, R. L.; Brinton, T. I.; Kile, D. E. Water solubility enhancement of some organic pollutants and pesticides by dissolved humic and fulvic acids. Environ. Sci. Technol. 1986, 20 (5), 502-508.

(8) Frimmel, F. H. Characterization of natural organic matter as major constituents in aquatic systems. J. Contam. Hydrol. 1998, 35 (13), 201-216.

(9) Rav-Acha, C.; Rebhun, M. Binding of organic solutes to dissolved humic substances and its effects on adsorption and transport in the aquatic environment. Water Res. 1992, 26 (12), 1645-1654.

(10) Chen, G.; Lin, C.; Chen, L.; Yang, H. Effect of size-fractionation dissolved organic matter on the mobility of prometryne in soil. Chemosphere 2010, 79 (11), 1046-1055.

(11) Burkhard, L. P. Estimating Dissolved Organic Carbon Partition Coefficients for Nonionic Organic Chemicals. Environ. Sci. Technol. 2000, 34 (22), 4663-4668.

(12) Flores-Céspedes, F.; Fernández-Pérez, M.; Villafranca-Sánchez, M.; González-Pradas, E. Cosorption study of organic pollutants and dissolved organic matter in a soil. Environ. Pollut. 2006, 142 (3), 449456.

(13) Ilani, T.; Schulz, E.; Chefetz, B. Interactions of Organic Compounds with Wastewater Dissolved Organic Matter: Role of Hydrophobic Fractions. J. Environ. Qual. 2005, 34 (2), 552-562.

(14) Williams, C. F.; Letey, J.; Farmer, W. J. Estimating the Potential for Facilitated Transport of Napropamide by Dissolved Organic Matter. Soil Sci. Soc. Am. J. 2005, 70 (1), 24-30.

(15) Sharma, S. K. Adsorptive Iron Removal from Groundwater; CRC Press: 2001; p 202.

(16) Zadaka, D.; Nir, S.; Radian, A.; Mishael, Y. G. Atrazine removal from water by polycation-clay composites: Effect of dissolved organic matter and comparison to activated carbon. Water Res. 2009, 43 (3), 677-683.

(17) Ernst, M.; Sachse, A.; Steinberg, C. W. E.; Jekel, M. Characterization of the DOC in nanofiltration permeates of a tertiary effluent. Water Res. 2000, 34, 2879-2886.

(18) Churchman, G. J. Formation of complexes between bentonite and different cationic polyelectrolytes and their use as sorbents for non-ionic and anionic pollutants. Appl. Clay Sci. 2002, 21 (3-4), 177189.

(19) Seckin, T.; Onal, Y.; Yesilada, O.; Gultek, A. Preparation and characterization of a clay-polyvinylpyridinium matrix for the removal of bacterial cells from water. J. Mater. Sci. 1997, 32, 5993-5999.

(20) Zhang, L. M.; Zhou, Y. J.; Wang, Y. Novel hydrogel composite for the removal of watersoluble cationic dye. J. Chem. Technol. Biotechnol. 2006, 81, 799-804.

(21) Ganigar, R.; Rytwo, G.; Gonen, Y.; Radian, A.; Mishael, Y. G. Polymer-clay nanocomposites for the removal of trichlorophenol and trinitrophenol from water. Appl. Clay Sci. 2010, 49 (3), 311-316.

(22) Radian, A.; Mishael, Y. G. Characterizing and Designing Polycation-Clay Nanocomposites As a Basis for Imazapyr Controlled Release Formulations. Environ. Sci. Technol. 2008, 42 (5), 1511-1516.

(23) Xu, H.; Wan, Y.; Li, H.; Zheng, S.; Zhu, D. Sorption of Aromatic Ionizable Organic Compounds to Montmorillonites Modified by Hexadecyltrimethyl Ammonium and Polydiallyldimethyl Ammonium. J. Environ. Qual. 2011, 40 (6), 1895-1902.

(24) Muller, S.; Totsche, K. U.; Kogel-Knabner, I. Sorption of polycyclic aromatic hydrocarbons to mineral surfaces. Eur. J. Soil Sci. 2007, 58 (4), 918-931.

(25) Zhu, D.; Herbert, B. E.; Schlautman, M. A.; Carraway, E. R.; J., H. Cation-pi Bonding: A New Perspective on the Sorption of Polycyclic Aromatic Hydrocarbons to Mineral Surfaces. J. Environ. Qual. 2004, 33, 1322-1330.

(26) Qu, X. L.; Liu, P.; Zhu, D. Q. Enhanced sorption of polycyclic aromatic hydrocarbons to tetra-alkyl ammonium modified smectites via cation-pi interactions. Environ. Sci. Technol. 2008, 42 (4), 11091116.

(27) Van Olphen, H.; Fripiat, J. J. Data Handbook for Clay Materials and Other Non-Metalic Minerals; Pergamon Press: Oxford, England, 1979.

(28) Gauthier, T. D.; Shane, E. C.; Guerin, W. F.; Seitz, W. R.; Grant, C. L. Fluorescence quenching method for determining equilibrium constants for polycyclic aromatic hydrocarbons binding to dissolved humic materials. Environ. Sci. Technol. 1986, 20 (11), 1162-1166.

(29) Parker, C. A. Photoluminescence of solutions; Amsterdam, 1968.

(30) Zadaka, D.; Radian, A.; Mishael, Y. G. Applying zeta potential measurements to characterize the adsorption on montmorillonite of organic cations as monomers, micelles, or polymers. J. Colloid Interface Sci. 2010, 352 (1), 171-177.

(31) Jung, A. V.; Frochot, C.; Villieras, F.; Lartiges, B. S.; Parant, S.; Viriot, M. L.; Bersillon, J. L. Interaction of pyrene fluoroprobe with natural and synthetic humic substances: Examining the local molecular organization from photophysical and interfacial processes. Chemosphere 2010, 80 (3), 228-234.

(32) Polubesova, T.; Sherman-Nakache, M.; Chefetz, B. Binding of Pyrene to Hydrophobic Fractions of Dissolved Organic Matter: Effect of Polyvalent Metal Complexation. Environ. Sci. Technol. 2007, 41 (15), 5389-5394. 
(33) Samios, S.; Lekkas, T.; Nikolaou, A.; Golfinopoulos, S. Structural investigations of aquatic humic substances from different watersheds. Desalination 2007, 210 (1-3), 125-137.

(34) Stevenson, F. J.; Goh, K. M. Infrared spectra of humic acids and related substances. Geochim. Cosmochim. Acta 1971, 35 (5), 471-483.

(35) Colthup, N. B. Spectra-Structure Correlation in the Infra-Red Region. J. Opt. Soc. Am. 1950, 40, 397-400.

(36) Bleiman, N.; Mishael, Y. G. Selenium removal from drinking water by adsorption to chitosan-clay composites and oxides: Batch and columns tests. J. Hazard. Mater. 2010, 183 (1-3), 590-595.

(37) Darder, M.; Colilla, M.; Ruiz-Hitzky, E. Chitosan-clay nanocomposites: application as electrochemical sensors. Appl. Clay Sci. 2005, 28 (1-4), 199-208.

(38) He, Y. Y.; Wang, X. C. Adsorption of a typical polycyclic aromatic hydrocarbon by humic substances in water and the effect of coexisting metal ions. Colloids Surf., A 2011, 379 (1-3), 93-101.

(39) Hwang, S. C.; Cutright, T. J. Impact of clay minerals and DOM on the competitive sorption/desorption of PAHs. Soil. Sediment. Contam. 2002, 11 (2), 269-291.

(40) Karimi-Lotfabad, S.; Pickard, M. A.; Gray, M. R. Reactions of Polynuclear Aromatic Hydrocarbons on Soil. Environ. Sci. Technol. 1996, 30 (4), 1145-1151.

(41) Liu, J. J.; Wang, X. C.; Fan, B. Characteristics of PAHs adsorption on inorganic particles and activated sludge in domestic wastewater treatment. Bioresour. Technol. 2011, 102 (9), 5305-5311.

(42) Wang, K.; Xing, B. Structural and Sorption Characteristics of Adsorbed Humic Acid on Clay Minerals. J. Environ. Qual. 2005, 34 (1), 342-349.

(43) Sharma, B.; Gardner, K. H.; Melton, J.; Hawkins, A.; Tracey, G. Evaluation of Activated Carbon as a Reactive Cap Sorbent for Sequestration of Polychlorinated Biphenyls in the Presence of Humic Acid. Environ. Eng. Sci. 2009, 26 (9), 1371-1379. 